\title{
MiR-320a acts as a prognostic factor and Inhibits metastasis of salivary adenoid cystic carcinoma by targeting ITGB3
}

\author{
Lijuan Sun ${ }^{1,2,3+}$, Bodu Liu ${ }^{1,3+}$, Zhaoyu Lin ${ }^{1,2+}$, Yandan Yao ${ }^{1,3}$, Yanyang Chen ${ }^{4}$, Yang Li ${ }^{4}$, Jianing Chen ${ }^{1,3}$, \\ Dongsheng $\mathrm{Yu}^{5}$, Zhangui Tang ${ }^{6}$, Bosheng Wang ${ }^{6}$, Shuguang Zeng ${ }^{7}$, Song Fan ${ }^{1,2}$, Youyuan Wang ${ }^{1,2}$, Yilin Li ${ }^{8}$, \\ Erwei Song ${ }^{1,3,9^{*}}$ and Jinsong Li $\mathrm{Li}^{1,2,9^{*}}$
}

\begin{abstract}
Background: Salivary Adenoid cystic carcinoma (SACC) patients with local invasion and lung metastasis are often resistant to conventional therapy such as operation, chemotherapy and radiotherapy. To explore the underling mechanisms, we studied the roles of miRNA in regulating invasiveness of SACC cells.

Methods: MicroRNA profiling was done in SACC cells with microarray. MiRNA mimics or antisense oligonucleotide was transfected and invasiveness of SACC cells was evaluated by adhesion assay and transwell assay. The target gene of miRNA was identified by luciferase reporter assay and "rescue" experiment. Tumor metastasis was evaluated by BALB/c-nu mice xenografts. MiRNA and its target gene expression were identified by in-situ hybridization and immunohistochemistry respectively, in 302 patients from affiliated hospitals of Sun Yat-sen University and in 148 patients from affiliated hospitals of Central South University, and correlated to the clinicopathological status of the patients.

Results: MiR-320a was down-regulated in high lung metastatic ACCM and SACC-LM cells compared with the corresponding low metastatic ACC2 and SACC-83 cells, and inhibited adhesion, invasion and migration of SACC cells by targeting integrin beta 3 (ITGB3). In vivo, enforced miR-320a expression suppressed metastasis of SACC xenografts. In the two independent sets, miR-320a was downregulated in primary SACCs with metastasis compared to those without metastasis, and low expression of this miRNA predicts poor patient survival and rapid metastasis. Multivariate analysis showed that miR-320a expression was an independent indicator of lung metastasis.
\end{abstract}

Conclusions: MiR-320a inhibits metastasis in SACCs by targeting ITGB3 and may serve as a therapeutic target and prognostic marker in salivary cancers.

Keywords: Metastasis, miR-320a, ITGB3, Adenoid cystic carcinoma, Prognosis

\section{Background}

Salivary adenoid cystic carcinoma (SACC) mainly occurs in the salivary duct, accounting for $24 \%$ of malignant salivary gland tumors. SACC exhibits certain unique characteristics, such as neurotropic, infiltrative growth and distant metastasis [1-3]. For SACC patients, distant metastasis is a crucial prognostic factor $[4,5]$. Therefore, determining the mechanisms that govern the perineural

\footnotetext{
*Correspondence: songerwei02@aliyun.com; lijinsong1967@163.com

${ }^{\dagger}$ Equal contributors

${ }^{1}$ Guangdong Provincial Key Laboratory of Malignant Tumor Epigenetics and Gene Regulation, Sun Yat-Sen Memorial Hospital, Sun Yat-Sen University, Guangzhou 510120, China

Full list of author information is available at the end of the article
}

invasion and metastasis of SACCs is essential for the development of novel therapeutic strategies to improve patient survival.

MicroRNAs (miRNAs) are a class of small non-coding RNAs ( 22 nucleotides in length) that are endogenously expressed in mammalian cells. miRNAs regulate gene expression by repressing mRNA translation or cleaving target mRNAs. miRNAs may function as oncogenes or tumor suppressors by modulating multiple cellular pathways, including cell proliferation [6], differentiation $[7,8]$, apoptosis $[9,10]$, and invasion [11-13]. There are significant differences in the miRNA expression profiles of a variety of human cancers, including colon, breast, 
lung, and stomach cancer, as well as lymphoma and leukemia, compared with the corresponding normal tissues $[6,7,14]$. Recently, a study found that 19 miRNAs were upregulated and 36 miRNAs were downregulated in primary SACC specimens, compared with matched normal samples [15]. However, the mechanisms by which miRNAs regulate the biological characteristics of SACC remains unclear.

Integrins are a family of transmembrane receptors that mediate the attachment between a cell and its surroundings, such as other cells or the extracellular matrix (ECM), and play important roles in signal transduction [16-19]. Integrins regulate cell proliferation, survival and migration mainly through FAK (focal adhesion kinase) and Src kinase family members [20,21]. Functional integrins are heterodimers containing two distinct subunits, $\alpha$ and $\beta$. There are $18 \alpha$ subunits and $8 \beta$ subunits found in mammals. Heterodimers formed between different subunits have different structures and functions $[17,18]$. It has been reported in breast, colorectal cancer and hepatocellular carcinoma that dysfunctions in microRNAregulated integrin signaling were involved in tumor metastasis and apoptosis [10,22,23].

In the present study, we screened for differentially expressed miRNAs in high lung metastatic SACC cells compared with their corresponding low metastatic cells and identified miR-320a as a metastatic repressor. We then explored the mechanism by which miR-320a regulates the invasiveness of SACC cells and the metastasis of SACC xenografts and identified its functional target as integrin beta 3 (ITGB3). Finally, we found significant correlations between miR-320a expression and the clinicopathological status and prognosis of SACC patients in two independent sets. These findings have advanced our knowledge of the molecular mechanisms of SACC metastasis and provided potential markers and therapeutic targets for the diagnosis and treatment of SACC.

\section{Results}

Reduction of miR-320a promotes invasiveness of salivary adenoid cystic carcinoma cells

To identify miRNAs involved in SACC metastasis, we screened for miRNAs that are differentially expressed in high lung metastatic ACCM cells and the parental low metastatic ACC2 cells via microarray analysis (Additional file 1). qRT-PCR further validated miR-320a as the most significantly downregulated miRNA in ACCM cells (Additional file 2). Transfection of the high lung metastatic cells with miR-320a mimics but not the non-relevant lin4 mimics specifically increased miR-320a levels in ACCM and SACC-LM cell $(P<0.01$, Figure $1 \mathrm{~A})$.

We next investigated whether ectopic expression of miR-320a inhibited the invasiveness in SACC cells. Transfection of miR-320a mimics remarkably inhibited the adhesion of ACCM cells (Figure $1 \mathrm{~B}$ and E, $P<0.05$ ). Transwell assays also demonstrated that miR-320a inhibited ACCM cell invasion and migration by $72 \%$ (Figure $1 \mathrm{C}$ and E, $P<0.05$ ) and $60 \%$ (Figure $1 \mathrm{D}$ and $\mathrm{E}, P<0.05$ ), respectively. Transfection with $\mathrm{miR}-320 \mathrm{a}$ also inhibited SACC-LM cell adhesion, invasion and migration by $75 \%$ (Figure $1 \mathrm{~B}$ and $\mathrm{E}, P=0.001$ ), $80 \%$ (Figure $1 \mathrm{C}$ and $\mathrm{E}, P=$ 0.008 ) and $82 \%$ (Figure $1 \mathrm{D}$ and E, $P=0.007$ ), respectively.

In contrast, silencing miR-320a expression in the parental ACC2 and SACC-83 cells using antisense oligonucleotides (ASO) enhanced the invasiveness of these cells (Additional file 3). The adhesion, invasion and migration of ACC2 cells increased by 2-, 3-, and 2.9-fold, respectively, and that of SACC-83 cells increased by 2.2-, 4.7- and 7.2-fold, respectively. Therefore, reduced miR-320a levels contribute to enhanced invasiveness in SACC cells.

\section{miR-320a inhibits the invasiveness of SACC cells by targeting ITGB3}

miRNAs act by binding to the 3 '-untranslated region (3'-UTR) of target genes through partial sequence homology. We predicted the target genes of miR-320a using TargetScan and found an miR-320a recognition site in the 3'-UTR of ITGB3 (Figure 2A). To confirm whether ITGB3 is a target of miR-320a, we performed luciferase reporter assays and evaluated the relative luciferase activities in SACC cells transfected with a reporter plasmid carrying an miR-320a target sequence (ITGB3 3'-UTR) versus those transfected with a control plasmid. It was shown that co-transfection with miR-320a mimics significantly reduced the luciferase activity in ACCM and SACC-LM cells (Figure 2B). However, when the miRNA target sequence was mutated in the reporter plasmids (ITGB3 3'-UTR mut), transfection with miR-320a mimics failed to influence the relative luciferase activity, suggesting that miR-320a suppresses ITGB3 expression in SACC cells.

To study whether ITGB3 serves as a functional target of miR-320a, we examined integrin $\beta 3$ expression in these cells. Western blot analysis showed that integrin $\beta 3$ expression in ACCM and SACC-LM cells was much higher than in the parental ACC2 and SACC-83 cells (Figure 2C). Transfection with miR-320a mimics reduced integrin $\beta 3$ expression in ACCM and SACC-LM cells, while transfection with the miR-320a ASO enhanced integrin $\beta 3$ expression in the parental ACC2 and SACC-83 cells. Furthermore, FAK and Src phosphorylation was suppressed by the miR-320a mimics, and enhanced by the miR-320a ASO, suggesting that miR-320a regulates the activity of the integrin signaling pathway. Furthermore, immunofluorescence staining demonstrated that the cytoskeletal protein actin accumulated in clusters and co-localized with clustered integrin $\beta 3$ on the ACCM cell surface (Figure 2D). These phenotypes were regulated 
A

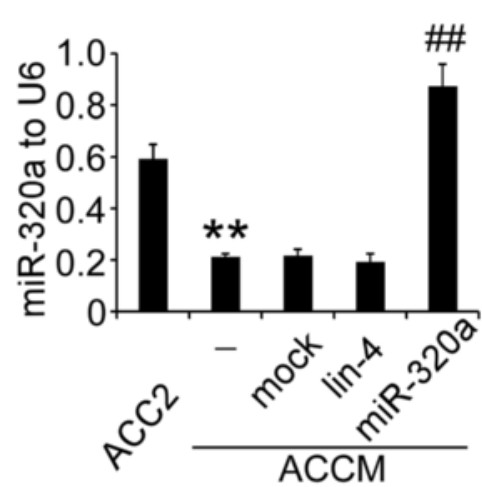

B

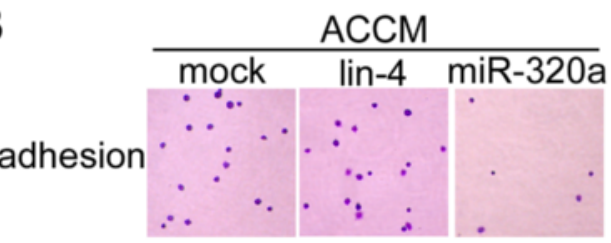

C

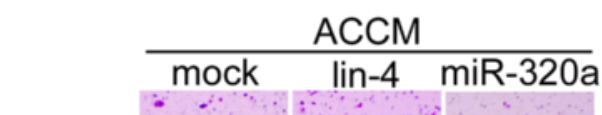

invasion
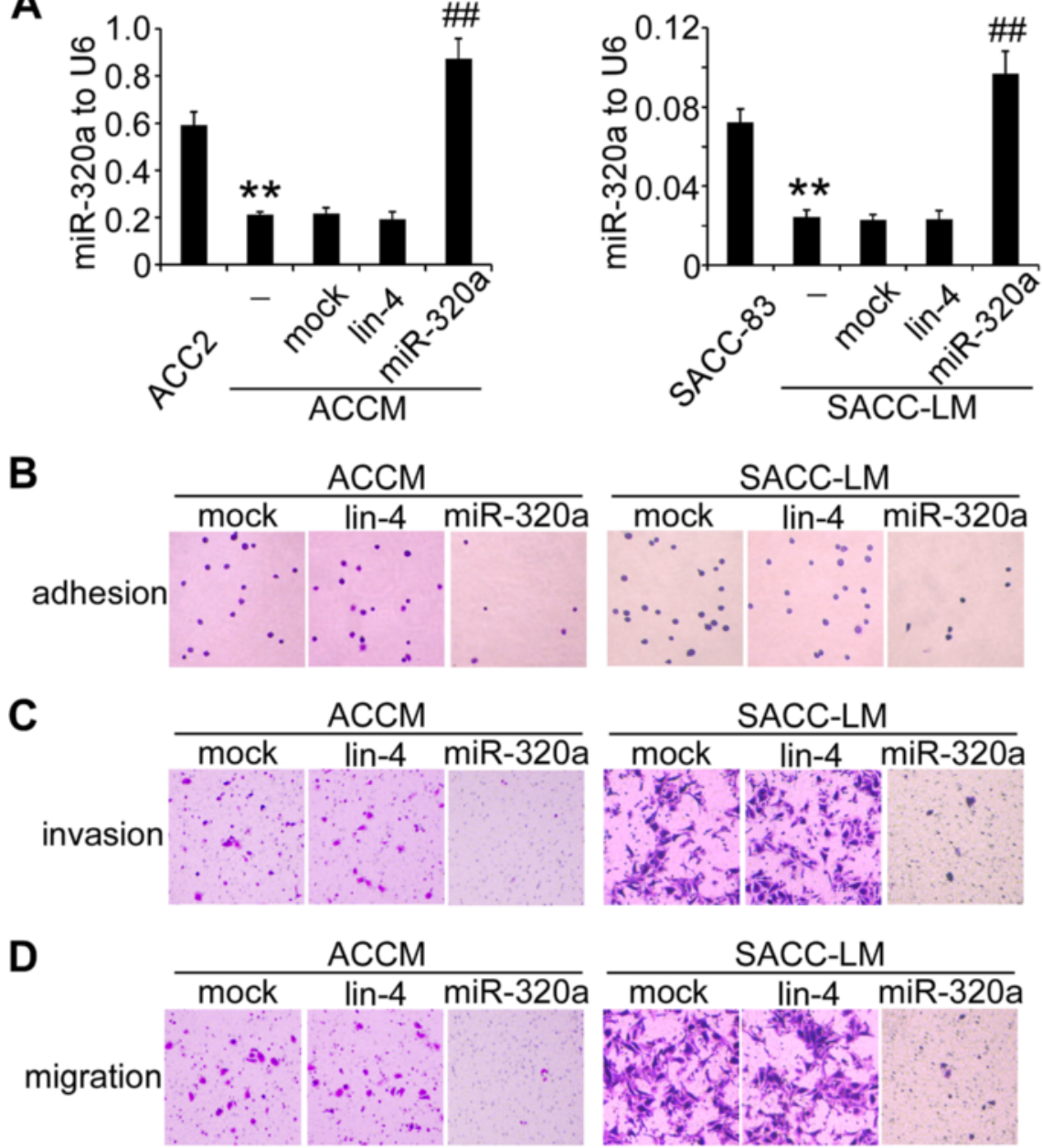

E
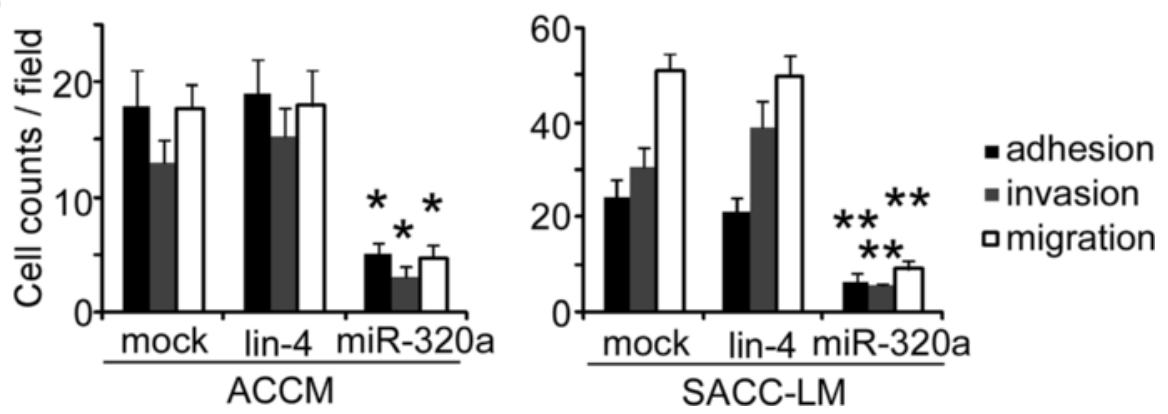

Figure 1 Ectopic expression of miR-320a inhibits the invasiveness of salivary adenoid cystic carcinoma cells. (A) miR-320a expression in SACC cells was determined by qRT-PCR analysis. ${ }^{* *} P<0.01$ vs. parental SACC cells. ${ }^{\# \#} P<0.01$ vs. mock transfection. U6 was used as an internal control. Adhesion assays (B) and transwell assays (C, D) showed that adhesion, invasion and migration of ACCM and SACC-LM cells were inhibited by miR-320a mimics (100X). (E) Quantification of adhesive, invasive and migratory cells assessed by adhesion assays and transwell assays. ${ }^{*} P<0.05$; ${ }^{* *} P<0.01$ vs. mock.

by miR-320a because transfecting ACCM cells with miR$320 \mathrm{a}$ mimics resulted in the downregulation of integrin $\beta 3$ and a ring-like distribution of actin. In contrast, in ACC2 cells, miR-320a ASO promoted the aggregation of actin and its co-localization with clustered integrin $\beta 3$ on the cell surface.
To identify whether miR-320a inhibits the invasiveness of SACC cells by targeting ITGB3, we performed a "rescue" experiment by co-transfecting ACCM cells with miR-320a mimics and a pcDNA3.1 vector that expresses ITGB3 with mutated miR-320a seed sequence (ITGB3 mut) in the $3^{\prime}$-UTR. Western blot analysis 


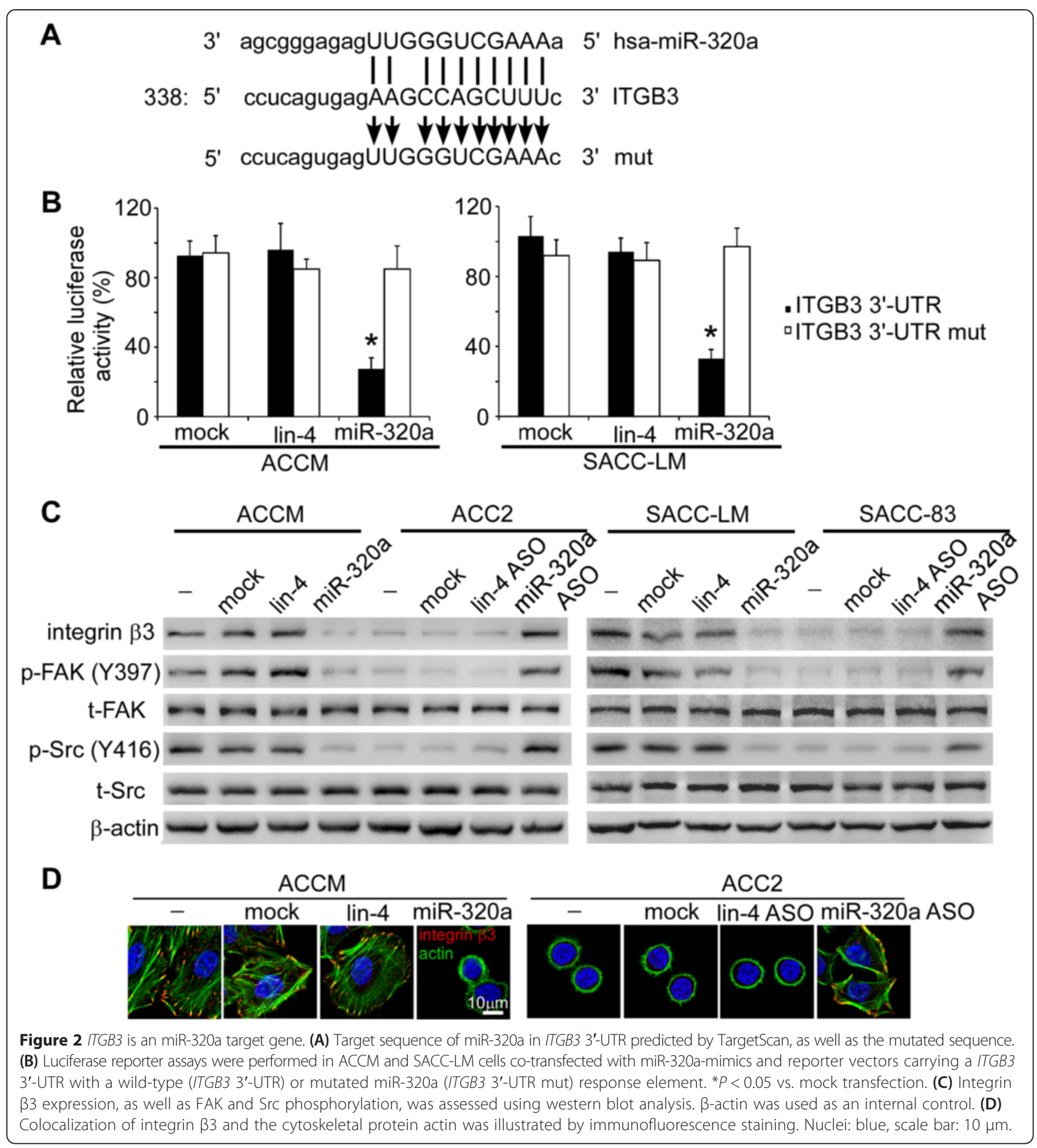

demonstrated that co-transfection of ACCM cells with miR-320a mimics and ITGB3 mut restored integrin $\beta 3$ expression, as well as FAK and Src phosphorylation, whereas co-transfecting wild-type pcDNA3.1-ITGB3 could not restore integrin $\beta 3$ expression after silencing by miR-320a mimics (Figure 3A). Moreover, "rescuing" integrin $\beta 3$ expression in the presence of miRNA mimics restored adhesion, invasion and migration of ACCM cells (Figure 3, B-E).
Collectively, these data suggest that miR-320a regulates the invasiveness of SACC cells by targeting ITGB3.

Enforced miR-320a expression inhibits ACCM xenograft metastasis

Given that miR-320a expression inhibits the invasiveness of SACC cells in vitro, we further assessed its effect on metastasis in vivo. miR-320a was stably expressed in ACCM 
A

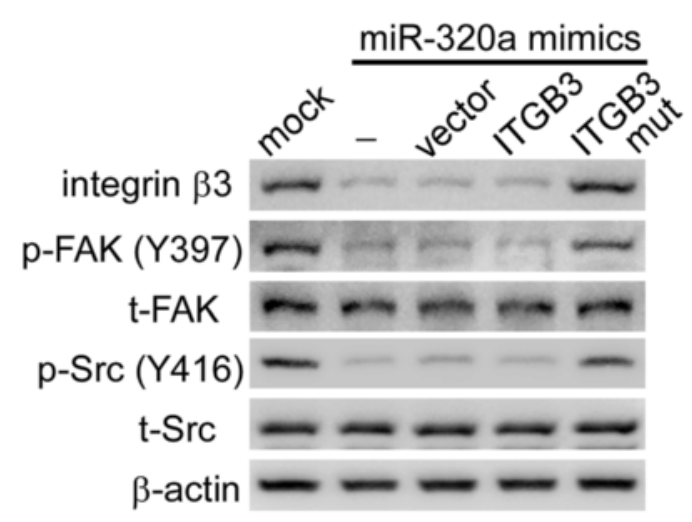

B

miR-320a mimics

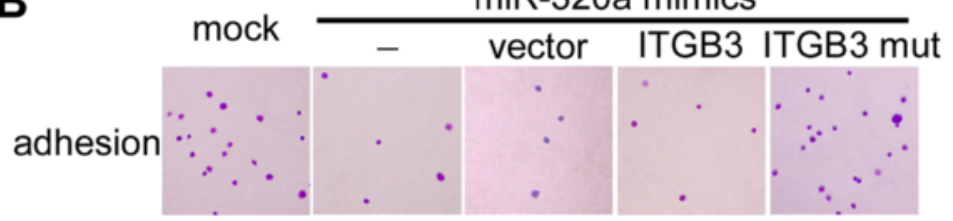

C

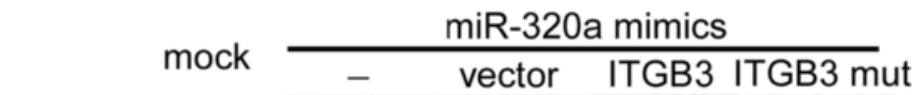

invasion

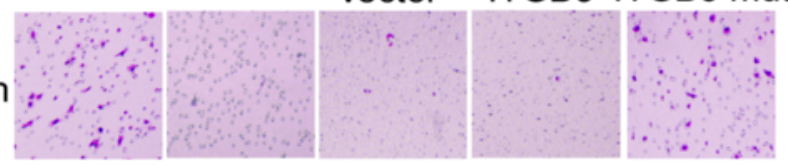

D

miR-320a mimics
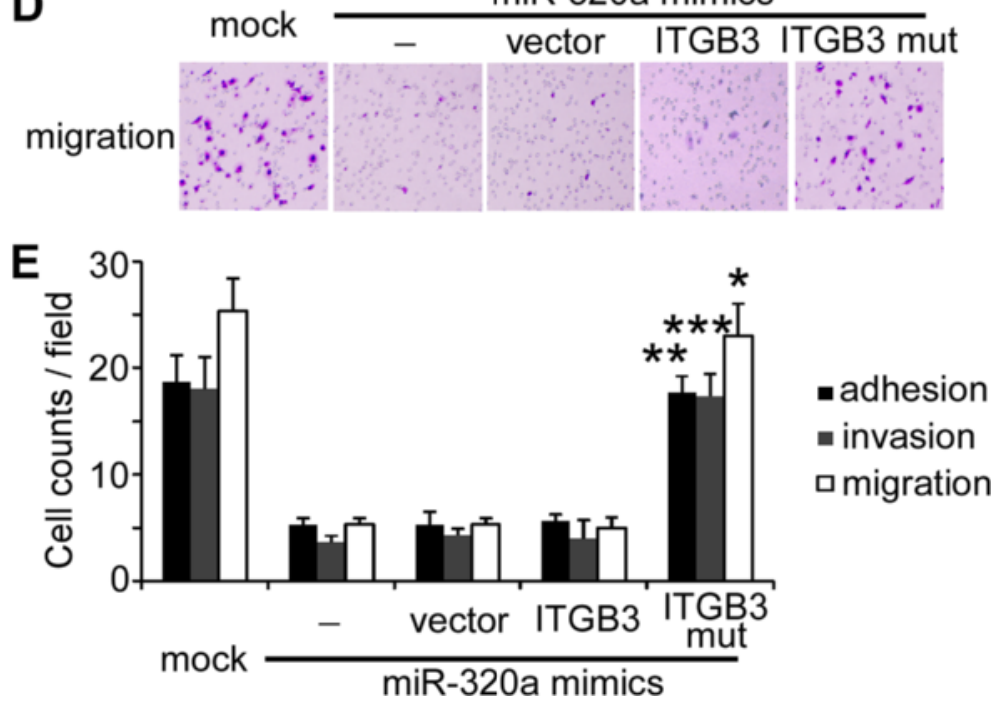

Figure 3 miR-320a inhibits the invasiveness of SACC cells by targeting ITGB3. (A) Integrin $\beta 3$ expression and FAK and Src phosphorylation were determined by western blot analysis in ACCM cells transfected with miR-320a mimics alone or in combination with either pcDNA 3.1+ (Vector) or pCDNA 3.1+ containing a wild-type (ITGB3) or mutant ITGB3 expression cassette of the miR-320a (ITGB3 mut) response element. $\beta$-actin was used as an internal control. Adhesion, invasion and migration of ACCM cells were assessed using adhesion assays (B) and transwell assays (C, $\mathbf{D})$ (100x). (E) Quantification of adhesive, invasive and migratory ACCM cells. ${ }^{*} P<0.05$; ${ }^{* *} P<0.01$; ${ }^{* *} P<0.001$ vs. vector.

cells by transduction with an miRNA-expressing vector (EmGFP-320a, Additional file 4A). As shown in Additional file 4B, enforced miR-320a expression did not alter tumor growth in BALB/c-nu mice inoculated with ACCM cells.
However, luminescence imaging of the whole body (Figure 4A) and harvested lungs and livers (Figure 4B) demonstrated that ACCM cells metastasized to the lungs and livers of tumor-bearing mice, and miR-320a expression 
A

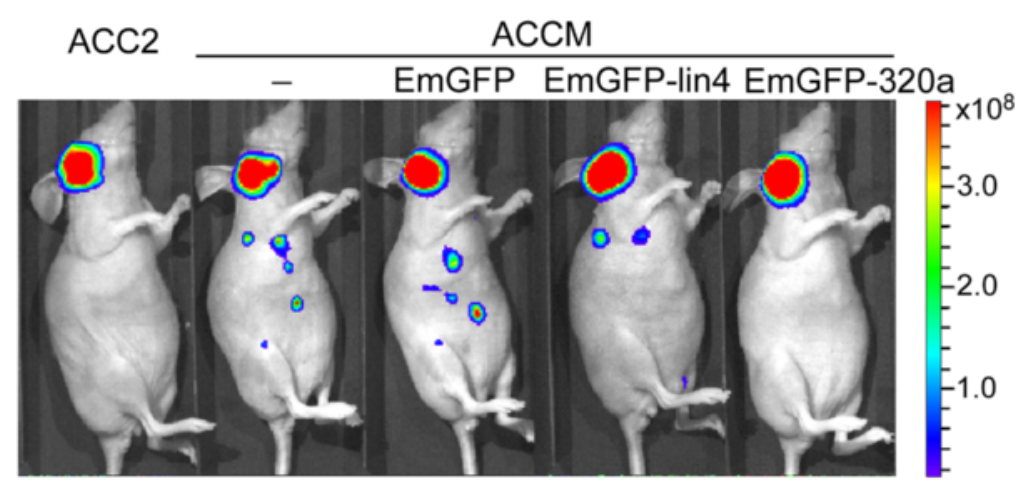

B

ACC2

ACCM
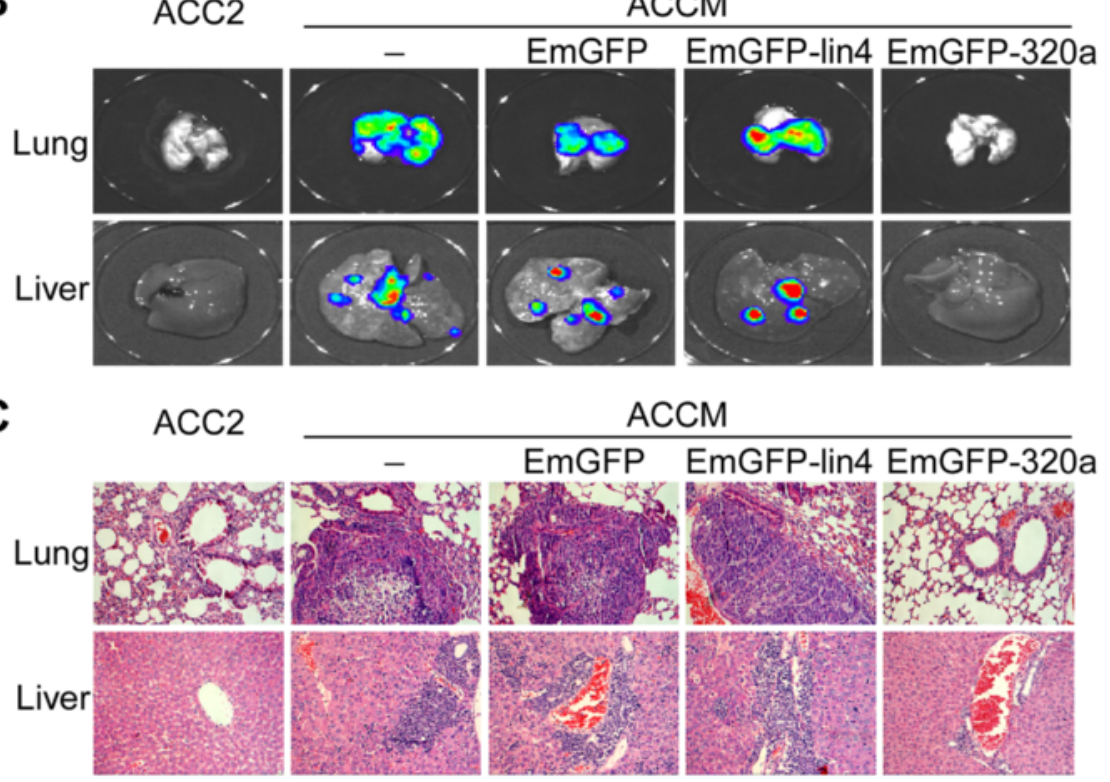

D
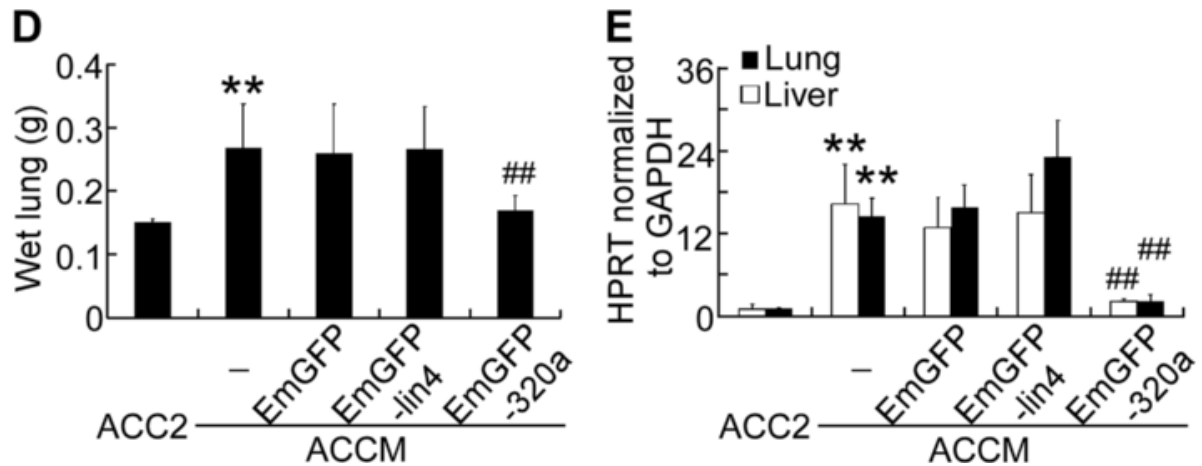

Figure 4 Ectopic expression of miR-320a inhibits ACCM xenograft metastasis in BALB/c-nu mice. (A) Whole-body luminal imaging was performed in mice inoculated with ACCM stably expressing the luciferase gene. Luminal images (B) and HE staining (C) of paraffin sections (200X) of the lungs (upper) and livers (lower) of the tumor-bearing mice. (D) Mean \pm SD wet lung weight of tumor-bearing mice ( $n=8 / g r o u p)$. (E) Expression of human HPRT mRNA relative to mouse $18 \mathrm{~S}$ rRNA in the lungs and livers of the tumor-bearing mice was determined by $q R T-P C R$ analysis. ${ }^{* *} P<0.01$ vs. ACC2 cells. ${ }^{\#} P<0.01$ vs. EmGFP transfection.

suppressed this metastasis. HE staining also revealed that miR-320a expression inhibited metastasis in the lungs and livers of mice bearing ACCM xenografts compared with non-relevant miRNA (Figure 4C). Furthermore, the average lung weight of ACCM tumor-bearing mice was reduced by miR-320a expression (Figure $4 \mathrm{D}, \mathrm{n}=8$, $P<0.01)$. The number of metastasized tumor cells, as quantified by qRT-PCR for human HPRT in tumorbearing mice, was reduced by $80 \%$ and $70 \%$ in the lungs and livers, respectively, of animals inoculated with miR-320a- 
expressing ACCM cells (Figure 4E, $P<0.01$ ). HE staining of the xenografts also indicated that the focal metastasis of ACCM tumors was markedly reduced by miR-320a (Additional file 4C). Therefore, miR-320a overexpression significantly suppressed the metastasis of ACCM xenografts.

Moreover, immunohistochemical staining revealed that miR-320a overexpression reduced integrin $\beta 3$ expression by more than $60 \%$ (Additional file $4 \mathrm{C}$ and $\mathrm{D}$ ) but did not influence the percentage of proliferative $\mathrm{PCNA}^{+}$tumor cells (Additional file $4 \mathrm{C}$ and E). These findings indicate that miR-320a inhibits SACC metastasis in vivo, most likely by silencing ITGB3.

\section{Low miR-320a expression indicates poor patient survival and high SACC metastasis}

We further evaluated the clinical significance of miR-320a expression in patient prognosis and SACC metastasis. In situ hybridization and immunohistochemical staining (Figure 5A) demonstrated that miR-320a expression was lower and integrin $\beta 3$ expression was higher in primary SACCs with metastasis compared with those without metastasis. The difference in expression between SACCs with metastasis and SACCs without metastasis from the affiliated hospitals of Sun Yat-sen University was statistically significant (Figure 5B, $P<0.001$ ). Spearman order correlation analysis showed that integrin $\beta 3$ expression in SACCs was inversely correlated with the miR-320a level (Additional file 5A, $r_{s}=-0.839, P<0.001$ ). Furthermore, SACCs with metastasis collected at affiliated hospitals of Central South University had remarkably low miR-320a expression and high integrin $\beta 3$ expression (Figure $5 \mathrm{C}, P<$ 0.001 ), and the integrin $\beta 3$ expression in the SACC paitents was negatively correlated with miR-320a levels (Additional file $\left.5 \mathrm{~B}, r_{s}=-0.857, P<0.001\right)$.

Next, we analyzed the association between miR-320a expression and the clinicopathologic status of SACC patients (Table 1). No significant correlation was observed between miR-320a expression and age, sex, tumor size or TNM stage. However, the miR-320a levels were closely associated with distant metastasis in patients from the two independent sets $(P<0.001$, or $P=0.001$, respectively $)$. Tumors with distant metastasis expressed low levels of miR-320a. Conversely, integrin $\beta 3$ expression was positively correlated with distant metastasis in SACC patients.

Furthermore, we generated Kaplan-Meier curves to evaluate the correlation between miR-320a expression and metastasis and the survival of the patients. The cumulative metastasis rate up to 10 years was significantly lower for patients with high miR-320a expression from the affiliated hospitals of Sun Yat-sen University than for those with low miR-320a expression (Figure 5D, 27.4\% vs. $52.4 \%, P<0.001)$. The cumulative survival rate was $62.8 \%$ for patients with high miR-320a expression, whereas it was only $44.4 \%$ for those with low miR-320a expression
(Figure $5 \mathrm{E}, P=0.001$ ). Similar results were obtained for patients from the affiliated hospitals of Central South University. The cumulative metastasis rate up to 10 years was $25.9 \%$ for patients with high miR-320a expression, as opposed to $55.9 \%$ for those with low miR-320a expression (Figure 5F, $P=0.001$ ), and the cumulative survival rate was $64.8 \%$ for patients with high miR-320a expression versus only $39.4 \%$ for those with low miR-320a expression (Figure 5G, $P=0.003$ ). In contrast, high integrin $\beta 3$ expression was associated with a high metastasis rate (Additional file 6A and $\mathrm{C}$ ) and poor survival in the patients (Additional file 6B and $\mathrm{D}$ ).

To determine whether miR-320a is an independent prognostic covariate for SACCs, we carried out a multivariate Cox proportional hazards analysis (Additional file 7). In the final multivariate Cox regression model, miR-320a expression in SACCs is associated with low lung metastasis (hazard ratio $[\mathrm{HR}]=0.45, P<0.001$ for patients from the affiliated hospitals of Sun Yat-sen University; $H R=0.363, P=0.001$ for patients from the affiliated hospitals of Central South University), independent of other clinical covariates, suggesting that miR-320a can be used as an independent indicator for lung metastasis in SACC patients.

\section{Discussion}

Dysregulation of miRNAs has been well documented in nearly all types of human malignancies, and numerous miRNAs are involved in tumor formation and progression by regulating the expression and action of many oncogenes and tumor suppressor genes. Previously, miR-320a was shown to be downregulated in colon cancer tissues and cancer cell lines, and ectopic expression of miR-320a suppressed the growth of colon cancer cells by directly targeting $\beta$-catenin [24]. In leukemia cells, enforced miR320 a expression suppresses transferrin receptor 1 expression and cell proliferation [25]. Furthermore, the miR-320a expression levels were significantly decreased in liver metastasis tissues compared with matched primary colorectal cancer tissues [26]. In our study, CCK8 assays and Annexin V/PI assays demonstrated that miR-320a expression did not influence the proliferation and apoptosis of SACC cells (data not shown). In vivo experiments also illustrated that miR-320a did not regulate tumor growth in ACC xenografts. However, reduced miR-320a expression is critical for the invasiveness of SACC cells, and ectopic miR-320a expression represses SACC tumor metastasis by silencing ITGB3. These results indicate that miR-320a primarily regulates the metastasis of SACCs. In addition, our study suggested that miR-320a exerts its anti-metastatic function by targeting ITGB3, a previously unidentified miR-320a target. This might explain the various functions of miR-320a among different cancer types while also suggesting that distinct features of SACC cells 


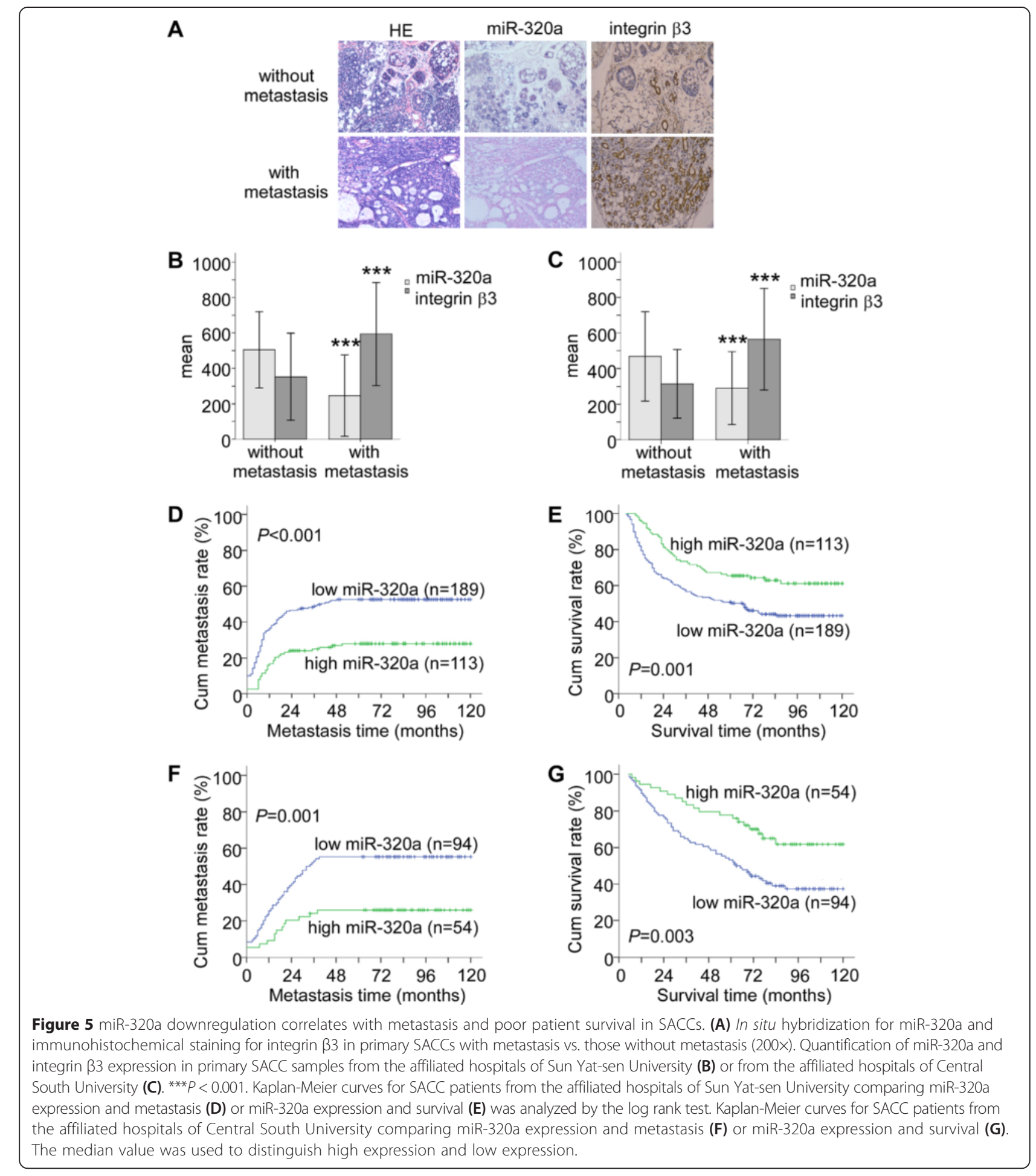

require careful consideration when developing a therapeutic strategy.

Local invasion and distant metastasis are the main causes of death in SACC patients. Therefore, determining the mechanisms that govern the metastasis of SACCs is essential for the development of novel therapeutic strategies to improve patient survival. In the present study, we found that ectopic miR-320a expression represses the invasiveness of SACC cells and the metastasis of SACC xenograft tumors, suggesting that miR-320a may be an effective therapeutic target. In contrast to artificially synthetic siRNAs, microRNAs are endogenous molecules that exist 
Table 1 Correlation among clinicopathologic status and the expression of miR-320a or integrin $\beta 3$ in SACC patients

A. Correlation among clinicopathologic status and the expression of miR-320a or integrin $\beta 3$ in SACC patients of Sun Yat-sen University

\begin{tabular}{llll}
\hline Characteristics & miR-320a (\%) & \\
\cline { 2 - 4 } & No. of low expression & No. of high expression & $P$ \\
\hline Sex & & 0.905
\end{tabular}

Integrin $\beta 3$ (\%)

No. of low expression $\quad$ No. of high expression

905

$45(33.6) \quad 89(66.4)$

106 (63.1)

62 (36.9)

73 (43.5)

95 (56.5)

Age

$<50$

82 (58.2)

59 (41.8)

107 (66.5)

54 (33.5)

0.153

54 (38.3)

64 (39.8)

0.801

Tumor Size

77 (38.1)

36 (36.0)

T3-4

125 (61.9)

64 (64.0)

103 (40.1)

10 (22.2)

35 (77.8)

(⿻)

69 (42.1)

44 (31.9)

94 (68.1)

$82(47.7)$

90 (52.3)

31 (23.8)

99 (76.2)

Status

Survival

84 (54.2)

71 (45.8)

105 (71.4)

42 (28.6)

83 (41.1)

35 (35.0)

0.029

107 (41.6)

11 (24.4)

0.074

Death
0.814

87 (61.7)

97 (60.2)

119 (58.9)

65 (65.0)

150 (58.4)

34 (75.6)

91 (55.5)

93 (67.4)

83 (53.5)

101 (68.7)

0.044

0.001

B. Correlation among clinicopathologic status and the expression of miR-320

Sex

Male

Female

$53(66.2)$

Age

$<50$

$\geq 50$

Tumor Size

T1-2

T3-4

Node metastasis

No

N1-N2

TNM stage

|-||

|II-IV

Distant Metastasis

No

Yes

Status

Survival

Death

47 (58.0)

47 (70.1)

60 (65.2)

34 (60.7)

80 (63.0)

$14(66.7)$

$56(66.7)$

38 (59.4)

42 (51.2)

52 (78.8)

37 (51.4)

57 (75.0)

27 (33.8)

27 (39.7)

34 (42.0)

20 (29.9)

32 (34.8)

22 (39.3)

47 (37.0)

7 (33.3)

28 (33.3)

26 (40.6)

40 (48.8)

14 (21.2)

35 (48.6)

19 (25.0)

$$
73 \text { (44.5) }
$$

45 (32.6)

$<0.001$
81 (47.1)
37 (28.5)
91 (52.9)
93 (71.5)

0.002
72(46.5)

46(31.3) 0.496

29 (36.2) 0.865

26 (38.2)

0.170

32 (39.5)

23 (34.3)

0.601

35 (38.0)

20 (35.7)

49 (38.6)

6 (28.6)

33 (39.3)

22 (34.4)

0.001

39 (47.6)

16 (24.2)

0.004
0.861

0.469

0.608

51 (63.8)

42 (61.8)

49 (60.5)

44 (65.7)

57 (62.0)

36 (64.3)

78 (61.4)

15 (71.4)

51 (60.7)

42 (65.6)

0.004

43 (52.4)

50 (75.8)

38 (52.8)

55 (72.4) 
in normal cells, which may minimize their unexpected off-target silencing effects $[27,28]$. In addition, because a microRNA molecule targets a set of coding genes rather than a single gene, therapies based on microRNA interference could enable more potent cancer treatments by targeting multiple molecular pathways.

In routine clinical practice, the TNM staging system is the key prognostic determinant for patients with salivary adenoid cystic carcinoma. However, large variations in the clinical outcomes of patients with the same cancer stage have been reported, suggesting that the present staging system is not adequate for prognosis. Here, we developed a miRNA signature that was predictive of metastasis in SACC patients, independent of TNM stage. TNM staging is performed mainly on the basis of anatomical information; conversely, the miRNA signature could show the biological characteristics of the SACCs. Identifying miRNAs in patients using quantitative RTPCR might be a straightforward and clinically applicable procedure. Furthermore, microRNAs are relatively stable compared with other biological macromolecules. miRNAs can be well preserved in tissue samples, even after formalin-fixation and paraffin-embedding, and they can be efficiently extracted and evaluated [29]. Moreover, microRNAs released from tumor cells are protected in membrane-derived exosomes and are thus stable in various bodily fluids, including serum and plasma. Alterations to the microRNA profile in many types of bodily fluids may reflect potential physiological and/or pathological conditions [30,31]. All specimens in our study were obtained from patients in China, and additional sets of independent samples from non-Asian patients will be needed to confirm our findings.

\section{Conclusions}

In summary, we found that miR-320a was downregulated in metastatic SACC cells, enabling the overexpression of its target ITGB3. Upregulated ITGB3 contributed to enhanced cell attachment, invasion and cancer metastasis. These results suggest that miR-320a is an effective metastasis inhibitor for SACC. We also identified a novel miR-320a target, ITGB3, in SACC cells, which may be a distinct feature of SACCs. Finally, miR-320a serves as a metastasis prediction marker independent of TNM staging. These findings provide a strong rationale for the potential use of miR-320a as a therapeutic target and prognostic biomarker. The findings of this study were summarized as an illustration (Additional file 8).

\section{Methods}

\section{Cell culture}

The human salivary adenoid cystic carcinoma cell lines ACC2 and ACCM were purchased from the Type Culture Collection of the Chinese Academy of Sciences (Shanghai,
China). SACC-83 and SACC-LM were purchased from Peking University (Beijing, China). ACCM and SACC-LM are highly metastatic cells derived from lung metastases of ACC2 and SACC-83 xenografts, respectively [32,33]. All cells were cultivated in RPMI-1640 medium (Gibco, Rockville, MD) supplemented with 10\% FBS (Invitrogen, Carlsbad, CA).

\section{MiRNA microarray analysis}

Microarray analyses were performed in ACC2 and ACCM cells as described previously [34]. A heat map demonstrating the average levels of microRNAs, which are differentially expressed in ACCM vs. ACC2, was created using DMVS 2.0 software (Chipscreen Biosciences, Shenzhen, China). The differentially expressed microRNAs are listed in Supplementary Table 1.

\section{Transfection}

All miRNA mimics and antisense oligonucleotides (ASOs) for miRNA were obtained from GenePharma (Shanghai, China). Cells were transfected with $30 \mathrm{nM}$ miRNA mimics or ASOs using Lipofectamine 2000 (Invitrogen). The EmGFP-miR-320a plasmid was also obtained from GenePharma, and blasticidin (Sigma, St Louis, MO) was used to select transfected ACCM cells. ITGB3 cDNA carrying a wild-type 3 '-UTR or a 3 '-UTR containing mutated seed sequence for miR-320a (ITGB3 mut) were cloned into pcDNA 3.1 for "rescue" experiments.

\section{Quantitative RT-PCR}

Real-time PCR was performed using a LightCycler 480 (Roche, Basel, Switzerland). Reactions were run in triplicate in three independent experiments. qRT-PCR for miRNA was performed using the Real-time PCR Universal Reagent (GenePharma). U6 was used as an internal control.

\section{Western blot analysis}

Protein extracts were resolved via 8\% SDS-PAGE, transferred onto polyvinylidene difluoride membranes (BioRad, Berkeley, CA), probed with antibodies against human integrin $\beta 3$ (Abcam, Cambridge, UK), p-FAK (Y397), FAK, p-Src (Y416), Src (Cell signaling, Boston, MA) or $\beta$-actin (Proteintech, Chicago, IL) followed by a peroxidaseconjugated secondary antibody (Proteintech), and then visualized by chemiluminescence (ImageQuant RT ECL, GE, Fairfield, CT).

\section{Adhesion assay}

Fibronectin-coated 24-well plates (Corning, New York, $\mathrm{NY}$ ) were seeded with, $1 \times 10^{4}$ cells/well and incubated for $15 \mathrm{~min}$, after which the non-adherent cells were washed away and the adherent cells were fixed in $4 \%$ paraformaldehyde, stained with crystal violet and counted (5 random $100 \times$ fields per well). Three independent experiments 
were performed, and the data are presented as the average \pm SD [35].

\section{Transwell assay}

A total of $1 \times 10^{5}$ cells were seeded into the upper chamber of a polycarbonate transwell filter chamber (Corning) and incubated for $22 \mathrm{~h}$. For the invasion assays, the upper chamber was coated with Basement Membrane (R\&D, Minneapolis, MN). Cells on the lower membrane surface were fixed in $4 \%$ paraformaldehyde, stained with crystal violet and counted (5 random 100 $\times$ fields per well). Three independent experiments were performed and the data are presented as the average $\pm \mathrm{SD}$.

\section{Luciferase reporter assay}

We cloned the miR-320a response element (wild type or mutant) of the 3'-UTR of ITGB3 into the pMIR-REPORT plasmid downstream of the luciferase reporter gene. Luciferase activity was assayed using a luciferase assay kit (Promega, Madison, WI), and the target effect was expressed as the relative luciferase activity of the reporter vector with the target sequence over that of the vector without the target sequence.

\section{Immunofluorescence staining}

Cells were stained for immunofluorescence on coverslips. After fixation and permeabilization, the cells were incubated with primary antibodies against integrin $\beta 3$ or FITC-phalloidin (Sigma) and then incubated with Alex 555-conjugated secondary antibodies (Invitrogen). The coverslips were counterstained with DAPI and imaged under a TCS SP5 confocal microscope (Leica, Solms, Germany).

\section{Tumor xenografts}

A total of $5 \times 10^{6}$ ACC2 or ACCM cells, either untransduced or transduced with the miRNA-expressing vector EmGFP-miR-320a, were injected into the salivary site of 5-week-old BALB/c-nu mice. After tumors were detected, the tumor size was measured and calculated: Volume $\left(\mathrm{mm}^{3}\right)=$ length $\times$ width $^{2} \times 0.5$. Tumor xenografts, as well as whole lung and liver tissues, were then harvested, weighed and snap-frozen in liquid nitrogen. To evaluate in vivo metastasis, fluorescence images of whole mice or their lungs and livers were acquired using an IVIS Lumina Imaging System (Xenogen, Alameda, CA), and portions of the lung and liver tissues were used for qRT-PCR for hHPRT (human hypoxanthine-guanine phosphoribosyltransferase) expression. Cryosections $(4 \mu \mathrm{m})$ were stained with hematoxylin and eosin (HE) and used for immunohistochemistry. The procedures were approved by Sun Yat-sen University Animal Care and Use Committee.

\section{Patients and tissue samples}

Two independent retrospective SACC patient cohorts were studied, including 302 patients from the affiliated hospitals of Sun Yat-sen University, such as Sun Yat-sen Memorial Hospital, the First Affiliated Hospital and the Hospital of Stomatology (Guangzhou, China), and 148 patients from the affiliated hospitals of Central South University, such as Xiangya Stomatological Hospital, the Second Xiangya Hospital (Changsha, China) between Jan 1, 1999, and Dec 31, 2008. None of the patients received any chemotherapy or radiotherapy prior to surgery. The tumors were staged according to the TNM staging system. The tissues were obtained from the respective pathology departments, and histological diagnosis and scoring of all the cases were performed by two independent pathologists (Yanyang Chen and Yang Li). Survival time was calculated from the date of surgery to the date of death or to the last follow-up. The date of death was obtained from patient records or through follow-up telephone calls. This study was approved by the institutional ethical review boards of both hospitals, and written informed consent was obtained from all patients.

\section{In situ hybridization}

This assay was performed according to the manufacturer's protocol (Exiqon, Vedbaek, Denmark). Briefly, after demasking, microRNA was hybridized to 5'-DIG-labeled $\mathrm{LNA}^{\text {tw }}$ probes. Then, the digoxigenin was recognized by a specific anti-DIG antibody that is directly conjugated to alkaline phosphatase. The nuclei were counterstained with hematoxylin.

\section{Immunohistochemistry}

For immunohistochemistry [36], the samples were incubated with an integrin $\beta 3$ antibody at $4^{\circ} \mathrm{C}$ overnight. The sections were then treated with a secondary antibody, followed by further incubation with a streptavidinhorseradish peroxidase complex. Diaminobenzidine (Dako, Carpinteria, CA) was used as a chromogen, and the nuclei were counterstained with hematoxylin.

\section{Scoring of ISH and IHC}

In each section, 5 fields of 200 tumor cells were counted randomly, and the scores for miR-320a and integrin $\beta 3$ were determined by combining the proportion of positively stained tumor cells and the intensity of staining. The proportion of positively stained tumor cells was graded from 0 to $3(0,<5 \%$ positive cells; $1,5-25 \%$; 2 , $26-50 \% ; 3,>50 \%)$. The intensity of staining was recorded on a scale of 0 (no staining), 1 (weak staining, light blue or yellow), 2 (moderate staining, blue or yellow), and 3 (strong staining, dark blue or yellow). For tumors that showed heterogeneous staining, the predominant pattern was taken into account for scoring. The staining index 
(SI) was calculated as follows: staining index $=$ proportion of positively stained tumor cells $\times$ staining intensity. Using this method, the expression of miR-320a and integrin $\beta 3$ was evaluated by the SI and scored as $0,1,2,3,4,6$, or 9 . A composite score greater than the median value was considered to be high expression, and composite scores less than or equal to the median value were considered to be low expression.

\section{Statistical analysis}

All statistical analyses were performed using SPSS 17.0 software for Windows (SPSS Inc., Chicago, IL). The Chisquared test was used to analyze the relationship between miR-320a or integrin $\beta 3$ expression and clinicopathologic characteristics. To measure the association between pairs of variables, Spearman order correlations were run. Kaplan-Meier survival curves were plotted, and the logrank test was performed. The significance of various variables for lung metastasis was analyzed by the Cox proportional hazards model in a multivariate analysis. All experiments with cell cultures were performed at least in triplicate. The results are expressed as the mean $\pm \mathrm{SD}$. $P<0.05$ was considered statistically significant.

\section{Additional files}

\section{Additional file 1: MicroRNA microarray analysis comparing ACC2 and ACCM cells.}

Additional file 2: The differential expression of miRNAs in the ACCM and ACC2 cells was validated using qRT-PCR analysis. U6 was used as an internal control.

Additional file 3: Reduction of miR-320a promotes the invasiveness of SACC cells. (A) miR-320a expression in SACC cells was determined using qRT-PCR analysis. ${ }^{*} P<0.05 ;{ }^{*} P<0.01$ vs. mock transfection. U6 was used as an internal control. An adhesion assay (B) and transwell assay (C, D) showed that the adhesion, invasion and migration of ACC2 and SACC-83 cells were enhanced by miR-320a ASO (100x). (E) Quantification of the adhesive, invasive and migratory cells assessed using the adhesion and transwell assays. ${ }^{*} P<0.05 ;{ }^{* *} P<0.01$ vs. mock.

Additional file 4: MiR-320a suppresses integrin $\beta 3$ expression in ACCM cells implanted in BALB/c-nu mice. (A) Transfection with the EmGFP-320a vector specifically enhances miR-320a expression in ACCM cells as determined by qRT-PCR. (B) Tumor volumes of mice inoculated with ACC2 cells or ACCM cells stably expressing miR-320a. (C) HE staining (200x) of the tumor and immunohistochemical staining (400x) for integrin $\beta 3$ and PCNA. The white arrows indicate focal metastases. The percentages of integrin $\beta 3$ (D) and PCNA (E) positive cells in the tumor section are analyzed. ${ }^{* *} P<0.01$ vs. ACC2 cells. ${ }^{\# \#} P<0.01$ vs. EmGFP transfection.

Additional file 5: MiR-320a expression was negatively correlated with integrin $\beta 3$ expression in SACCs. Associations between miR-320a expression and integrin $\beta 3$ expression in SACC samples from affiliated hospitals of Sun Yat-sen University (A) or from affiliated hospitals of Central South University (B) were analyzed using Spearman's rank order correlation coefficient.

Additional file 6: High expression of integrin $\beta 3$ indicates poor patient survival and high risk of SACC metastasis. Kaplan-Meier curves for SACC patients from affiliated hospitals of Sun Yat-sen University plotted according to integrin $\beta 3$ expression and metastasis difference (A) or plotted according to integrin $\beta 3$ expression and survival difference (B) were analyzed using the log rank test. Kaplan-Meier curves for SACC patients from affiliated hospitals of Central South University plotted according to integrin $\beta 3$ expression and metastasis difference $(C)$ or plotted according to integrin $\beta 3$ expression and survival difference (D). The median level was used to distinguish high expression and low expression.

Additional file 7: Multivariate analysis of various variables for lung metastasis in SACC patients using Cox regression analysis.

Additional file 8: Schematic summary of the findings of this study.

\section{Competing interests}

The authors declare that they have no competing interests.

\section{Authors' contributions}

LS and BL carried out the experiments, participated in the design of study, the analysis of the data, and drafted the manuscript. ZL and YY carried out the experiments, and analyzed the data. YC, YL, JC, ZT, BW and YL helped in acquisition of data. DY, SZ, SF and YW provided technical and material support. ES and $\mathrm{J}$ conceived the study, participated in its design and coordination, and helped to draft the manuscript. All authors read and approved the final manuscript.

\section{Acknowledgments}

This work was supported by National Natural Science Foundation of China (81272951 and 81072225 to J.L., 81272894, 81230060, 81261140373 to E.S., 81302369 to L.S.), by Specialized Research Fund for the Doctoral Program of Higher Education (20110171110068 to J.L.), by Science and Technology Project of Guangzhou City (11C22060035 to J.L.), by the Fundamental Research Funds for the Central Universities (13ykpy27 to L.S.), by Fund for Excellent Doctoral Dissertation of Guangdong Province (81000-3212502 to L.S), by Grant KLB09001 from the Key Laboratory of Malignant Tumor Gene Regulation and Target Therapy of Guangdong Higher Education Institutes, Sun Yat-Sen University, by Grant [2013] 163 from Key Laboratory of Malignant Tumor Molecular Mechanism and Translational Medicine of Guangzhou Bureau of Science and Information Technology.

\section{Author details}

${ }^{1}$ Guangdong Provincial Key Laboratory of Malignant Tumor Epigenetics and Gene Regulation, Sun Yat-Sen Memorial Hospital, Sun Yat-Sen University, Guangzhou 510120, China. ${ }^{2}$ Department of Oral \& Maxillofacial Surgery, Sun Yat-sen Memorial Hospital, Sun Yat-sen University, Guangzhou 510120, China. ${ }^{3}$ Breast Tumor Center, Sun Yat-sen Memorial Hospital, Sun Yat-sen University, Guangzhou 510120, China. ${ }^{4}$ Department of Pathology, the First Affiliated Hospital, Sun Yat-sen University, Guangzhou 510080, China. ${ }^{5}$ Guanghua School of Stomatology, Sun Yat-sen University, Guangzhou 510055, China. ${ }^{6}$ Xiangya School of Stomatology, Central South University, Changsha 410078, China. ${ }^{7}$ Guangdong Provincial Stomatological Hospital, Guangzhou 510280, China. ${ }^{8}$ Xaverian Brothers High School, Westwood, MA 02090, USA. ${ }^{9}$ Sun Yatsen Memorial Hospital, Sun Yat-sen University, Guangzhou 510120, China.

Received: 21 November 2014 Accepted: 17 March 2015

Published online: 29 April 2015

\section{References}

1. Dodd RL, Slevin NJ. Salivary gland adenoid cystic carcinoma: a review of chemotherapy and molecular therapies. Oral Oncol. 2006;42:759-69.

2. Szanto PA, Luna MA, Tortoledo ME, White RA. Histologic grading of adenoid cystic carcinoma of the salivary glands. Cancer. 1984;54:1062-9.

3. Tang Y, Liang X, Zheng M, Zhu Z, Zhu G, Yang J, et al. Expression of c-kit and Slug correlates with invasion and metastasis of salivary adenoid cystic carcinoma. Oral Oncol. 2010;46:311-6.

4. Gupta GP, Massague J. Cancer metastasis: building a framework. Cell. 2006;127:679-95.

5. Rapidis AD, Givalos N, Gakiopoulou H, Faratzis G, Stavrianos SD, Vilos GA, et al. Adenoid cystic carcinoma of the head and neck. Clinicopathological analysis of 23 patients and review of the literature. Oral Oncol. 2005;41:328-35.

6. Hayashita Y, Osada H, Tatematsu Y, Yamada H, Yanagisawa K, Tomida S, et al. A polycistronic microRNA cluster, miR-17-92, is overexpressed in human lung cancers and enhances cell proliferation. Cancer Res. 2005;65:9628-32.

7. Chen CZ, Li L, Lodish HF, Bartel DP. MicroRNAs modulate hematopoietic lineage differentiation. Science. 2004;303:83-6.

8. Yu F, Yao H, Zhu P, Zhang X, Pan Q, Gong C, et al. let-7 regulates self renewal and tumorigenicity of breast cancer cells. Cell. 2007;131:1109-23. 
9. Li J, Huang H, Sun L, Yang M, Pan C, Chen W, et al. MiR-21 indicates poor prognosis in tongue squamous cell carcinomas as an apoptosis inhibitor. Clin Cancer Res. 2009;15:3998-4008.

10. Yu F, Deng H, Yao H, Liu Q, Su F, Song E. Mir-30 reduction maintains selfrenewal and inhibits apoptosis in breast tumor-initiating cells. Oncogene. 2010;29:4194-204.

11. Wellner U, Schubert J, Burk UC, Schmalhofer O, Zhu F, Sonntag A, et al. The EMT-activator ZEB1 promotes tumorigenicity by repressing stemness-inhibiting microRNAs. Nat Cell Biol. 2009;11:1487-95.

12. Paterson EL, Kazenwadel J, Bert AG, Khew-Goodall Y, Ruszkiewicz A, Goodall GJ. Down-regulation of the miRNA-200 family at the invasive front of colorectal cancers with degraded basement membrane indicates EMT is involved in cancer progression. Neoplasia. 2013;15:180-91.

13. Liu MX, Zhou KC, Cao Y. MCRS1 overexpression, which is specifically inhibited by miR-129*, promotes the epithelial-mesenchymal transition and metastasis in non-small cell lung cancer. Mol Cancer. 2014;13:245.

14. Couzin J. Cancer biology. A new cancer player takes the stage. Science. 2005;310:766-7.

15. Mitani Y, Roberts DB, Fatani H, Weber RS, Kies MS, Lippman SM, et al. MicroRNA profiling of salivary adenoid cystic carcinoma: association of miR-17-92 upregulation with poor outcome. PLoS One. 2013:8:e66778.

16. Deng X, Li Q, Hoff J, Novak M, Yang H, Jin H, et al. Integrin-associated CD151 drives ErbB2-evoked mammary tumor onset and metastasis. Neoplasia. 2012;14:678-89.

17. Clark EA, Brugge JS. Integrins and signal transduction pathways: the road taken. Science. 1995;268:233-9.

18. Schwartz W, Schaller M, M. G. Integrins: emerging paradigms of signal transduction. Annu Rev Cell Dev Biol. 1995;11:549-99.

19. Hu Y, Ylivinkka I, Chen P, Li L, Hautaniemi S, Nyman TA, et al. Netrin-4 promotes glioblastoma cell proliferation through integrin beta4 signaling. Neoplasia. 2012;14:219-27.

20. Guan JL. Role of focal adhesion kinase in integrin signaling. Int J Biochem Cell Biol. 1997;29:1085-96.

21. Vuori K, Hirai H, Aizawa S, Rouslahti E. Introduction of p130cas signaling complex formation upon integrin-mediated cell adhesion: a role for Src family kinases. Mol Cell Biol. 1996;16:2606-13.

22. Zha RP, Guo WJ, Zhang ZF, Qiu ZP, Wang QF, Ding J, et al. Genome-Wide Screening Identified That miR-134 Acts as a Metastasis Suppressor by Targeting Integrin beta 1 in Hepatocellular Carcinoma. PLoS One. 2014;9:e87665.

23. Zhao YY, Miao G, Li Y, Isaji T, Gu JG, Li J, et al. Microrna 130b Suppresses Migration and Invasion of Colorectal Cancer Cells through Downregulation of Integrin beta 1. PLoS One. 2014;9:e87938.

24. Sun JY, Huang Y, Li JP, Zhang X, Wang L, Meng YL, et al. MicroRNA-320a suppresses human colon cancer cell proliferation by directly targeting beta-catenin. Biochem Biophys Res Commun. 2012;420:787-92.

25. Schaar DG, Medina DJ, Moore DF, Strair RK, Ting Y. miR-320 targets transferrin receptor 1 (CD71) and inhibits cell proliferation. Exp Hematol. 2009;37:245-55.

26. Zhang $Y$, He X, Liu Y, Ye Y, Zhang H, He P, et al. microRNA-320a inhibits tumor invasion by targeting neuropilin 1 and is associated with liver metastasis in colorectal cancer. Oncol Rep. 2012;27:685-94.

27. Krützfeldt J, Rajewsky N, Braich R, Rajeev KG, Tuschl T, Manoharan M, et al. Silencing of microRNAs in vivo with 'antagomirs'. Nature. 2005;438:685-9.

28. Jeyaseelan K, Herath WB, Armugam A. MicroRNAs as therapeutic targets in human diseases. Expert Opin Ther Targets. 2007;11:1119-29.

29. Li J, Smyth P, Flavin R, Cahill S, Denning K, Aherne S, et al. Comparison of miRNA expression patterns using total RNA extracted from matched samples of formalin-fixed paraffin-embedded (FFPE) cells and snap frozen cells. BMC Biotechnol. 2007;7:36.

30. Gilad S, Meiri E, Yogev Y, Benjamin S, Lebanony D, Yerushalmi N, et al. Serum microRNAs are promising novel biomarkers. PLoS One. 2008;3:e3148.

31. Mitchell PS, Parkin RK, Kroh EM, Fritz BR, Wyman SK, Pogosova-Agadjanyan EL, et al. Circulating microRNAs as stable blood-based markers for cancer detection. Proc Natl Acad Sci U S A. 2008;105:10513-8.

32. Guan X, Qiu W, He R, Lin G, X. Z. The selection of highly lung metastatic salivary cystic carcinoma clone. Chin J Stomatol. 1996;31:74-7.

33. Li S, Liu X, Zhang K. Establishment of a human cancer cell line from adenoid cystic carcinoma of the minor salivary gland. Chin J Stomatol. 1990;25:29-31.

34. Sun L, Yao Y, Liu B, Lin Z, Lin L, Yang M, et al. MiR-200b and miR-15b regulate chemotherapy-induced epithelial-mesenchymal transition in human tongue cancer cells by targeting BMI1. Oncogene. 2012;31:432-45.
35. Chen J, Yao Y, Gong C, Yu F, Su S, Chen J, et al. CCL18 from tumor-associated macrophages promotes breast cancer metastasis via PITPNM3. Cancer Cell. 2011;19:541-55.

36. Song E, Chen J, Ouyang N, Su F, Wang M, Heemann U. Soluble Fas ligand released by colon adenocarcinoma cells induces host lymphocyte apoptosis: an active mode of immune evasion in colon cancer. Br J Cancer. 2001;85:1047-54.

\section{Submit your next manuscript to BioMed Central and take full advantage of:}

- Convenient online submission

- Thorough peer review

- No space constraints or color figure charges

- Immediate publication on acceptance

- Inclusion in PubMed, CAS, Scopus and Google Scholar

- Research which is freely available for redistribution 